died in a hospital in the region but had no record of admission; they had probably been brought in dead or died soon after arrival at hospital without a formal admission. Ten had died at residential addresses. The median age of the 25 was 16 years, and 11 (44\%) were aged at least 20 years. Of all 146 people who died, 25 (17\%) died without admission to hospital, 41 (28\%) died on the day of admission, and 40 (27\%) died the day after admission.

Incidence has risen substantially in recent years (table). Case fatality rates have not significantly declined over time $(\mathrm{P}=0.31$ for cases admitted to hospital, and $\mathrm{P}=0.28$ for all cases). We found no significant trends in case fatality rates within individual age groups (see tables on bmj.com). For those admitted to hospital, case fatality rates at 30 days were similar in the teaching hospital $(8.9 \%$; $95 \%$ confidence interval $5.6 \%$ to $12.2 \%)$ and non-teaching hospitals $(9.4 \% ; 7.6 \%$ to $11.3 \%$ ), and in males and females.

\section{Comment}

Case fatality rates for meningococcal disease in the Oxford region have not fallen since the late 1960s. Others have also reported no recent reduction in case fatality rates in population based studies, ${ }^{1-3}$ although recent declines in a specialist paediatric unit have been described. ${ }^{4}$ In our study, a relatively high percentage of people who died outside hospital, or on arrival, were adults and perhaps had been less closely observed than children in their illness outside hospital. Our case fatality rate of $11 \%$ is the same as that found in a study based on multiple source case ascertainment and case note review in an adjacent health region in 1969-74. ${ }^{4}$ In that study, $22 \%$ of all deaths, compared to $17 \%$ in this study, occurred without admission to hospital or in people who were dead on arrival. ${ }^{5}$

The time from onset to death is usually rapid. This, and the fact that death rates have not declined, emphasises the need for vigilance in making the diagnosis and the importance of prevention through immunisation and, when appropriate, chemoprophylaxis for contacts.

Contributors: MJG designed the study and wrote the manuscript. SER did the analyses and wrote the manuscript. DY programmed the Oxford record linkage study database. MJG and SER are guarantors.

Funding: SER receives funding from the Department of Health as part of its funding for the National Centre for Health Outcomes Development; and the Unit of Health-Care Epidemiology is funded by the Research and Development Directorate of the Department of Health and Social Care (South). The views expressed in this paper are those of the authors and not necessarily those of the Department of Health. Competing interests: None declared.

1 Wylie PA, Stevens D, Drake W III, Stuart J, Cartwright K. Epidemiology and clinical management of meningococcal disease in west Gloucestershire: retrospective, population based study. BMJ 1997;315:774-9.

2 Sorensen HT, Steffensen FH, Schonheyder HC, Nielsen GL, Hansen I, Madsen KM, et al. Trend in incidence and case fatality of meningococcal disease over 16 years in northern Denmark. Eur J Clin Microbiol Infect Dis disease over 16 ye $17: 690-4$.

3 Booy R, Habibi P, Nadel S, de Munter C, Britto J, Morrison A, et al. Meningococcal Research Group. Reduction in case fatality rate from meningococcal disease associated with improved healthcare delivery. Arch Dis Child 2001;85:386-90.

4 Goldacre MJ. Acute bacterial meningitis in childhood: incidence and mortality in a defined population. Lancet 1976;1:28-31.

Goldacre MJ. Acute bacterial meningitis: where do children die? Int J Epidemiol 1976;5:343-7.

(Accepted 16June 2003)

\title{
Prophylaxis for venous thromboembolism during treatment for cancer: questionnaire survey
}

\author{
C C Kirwan, E Nath, G J Byrne, C N McCollum
}

Venous thromboembolism is common in patients with cancer and is often the cause of death. ${ }^{1}$ Patients receiving treatment for cancer are at even greater risk of thromboembolism. Thromboembolism occurs in 5\% of patients receiving chemotherapy for early breast carcinoma, ${ }^{2}$ and up to $17.6 \%$ of patients receiving chemotherapy for metastatic breast disease are affected. ${ }^{3}$ Patients with node-negative breast cancer taking tamoxifen were six times more likely to develop venous thromboembolism. ${ }^{4}$

Adjuvant use of tamoxifen carries a relative risk of 1.22 compared with no treatment. Combining methods of treatment further increases the risk of thromboembolism. Chemotherapy with tamoxifen increases risk by 3.5 times compared with chemotherapy alone, ${ }^{5}$ and preoperative radiotherapy for rectal carcinoma doubles the postoperative risk of venous thrombosis. ${ }^{5}$ Low doses (1 mg) of warfarin throughout chemotherapy for metastatic breast cancer are associated with a relative risk reduction of $85 \%$ with no increase in serious bleeding complications. ${ }^{1}$

\section{Participants, methods, and results}

We sent a postal questionnaire to all oncologists in northern England, identified by internet search and in the Medical Directory 2002. We used a scoring system to establish specialty, main type of cancer treated, main method of treatment (chemotherapy, hormone therapy, or radiotherapy), and current prophylaxis practice and estimate of risk of venous thrombembolism.

Of the 123 responses to the 166 questionnaires we sent, 106 (64\%) were acceptably completed. Half the oncologists (56) specialised in clinical oncology, 31 in medical oncology, seven in surgery, five in gynaecological oncology, five in paediatrics, one in urology, and one in radiology. We have no information about the specialties of oncologists who did not respond. The most common treatment was chemotherapy, used by $41(39 \%)$ oncologists; $10(9 \%)$ used hormone therapy and $44(42 \%)$ used radiotherapy. The oncologists treated many types of tumour. 
Factors that cause 106 oncologists in northern England to initiate use of prophylaxis*

\begin{tabular}{lc} 
Factor & $\begin{array}{c}\text { No of oncologists } \\
\text { altering practice }\end{array}$ \\
\hline Previous venous thromboembolism & 48 \\
\hline Immobility & 47 \\
\hline Thrombophilia & 25 \\
\hline Central venous line & 18 \\
\hline Obesity & 16 \\
\hline Surgery & 9 \\
\hline Concurrent hormone therapy & 8 \\
\hline Family history & 6 \\
\hline Pelvic mass & 3 \\
\hline Superior vena caval obstruction & 2 \\
\hline Age & 2 \\
\hline Brachytherapy & 1 \\
\hline Multiple myeloma & 1 \\
\hline Hyperviscosity & 1 \\
\hline Travel & 1 \\
\hline
\end{tabular}

${ }^{*} 19$ oncologists never use prophylaxis for venous thromboembolism.

A total of 29 (27.4\%; 95\% confidence interval 19.8\% to $36.5 \%$ ) oncologists thought their patients were not at risk of venous thromboembolism. This response was independent of the type of tumour treated. Considering different methods of treatment individually, 71 oncologists believed that hormone therapy posed little or no increased risk to patients; 83 thought the same for chemotherapy and 96 for radiotherapy.

Of the 106 oncologists, 84,79 , and 86 reported not using prophylaxis routinely in chemotherapy, hormone therapy, and radiotherapy. As risk factors, nine of the 10 oncologists who used prophylaxis in chemotherapy mentioned central venous lines and six of the 11 who used prophylaxis in hormone therapy mentioned stilboestrel. Preferred prophylaxis was aspirin (13), warfarin (12), subcutaneous heparin (10), stockings (9), and randomising patients with central venous lines to different warfarin regimens (3). Many factors cause oncologists to prescribe prophylaxis (table). A total of $19(17.9 \% ; 11.8$ to $26.3 \%)$ oncologists never used prophylaxis for venous thromboembolism.

Of those who answered, $37 \%$ of oncologists estimated that less than $1 \%$ of their patients werecurrently using prophylaxis for venous thrombo- embolism and 62\% estimated less than 5\%; 16\% did not answer this question.

\section{Comment}

More than a quarter of oncologists do not recognise the thrombogenic effects of treatments for cancer, and thromboprophylaxis is rarely used in patients undergoing treatment for cancer. Oncologists estimated that a surprisingly low percentage of patients were using prophylaxis bearing in mind that half of respondents mentioned previous venous thromboembolism and immobility as indications for routine prophylaxis. The response of a third of oncologists that venous thromboembolism does not pose a risk is not biased by lower risk specialties such as paediatrics. The good response rate to our questionnaire demonstrates a reliable representation of current practice in the north of England. National guidelines on prophylaxis for venous thromboembolism during cancer treatment are needed.

Contributors: CCK designed the questionnaire and analysed the responses. EN collected data and identified oncologists. GJB and NMMcC helped to design the questionnaire and write the paper. CCK is guarantor.

Funding: No additional funding.

Competing interests: None declared.

Ethical approval: Not needed.

1 Jilma B, Kamath S, Lip GYH. ABC of antithrombotic therapy: antithrombotic therapy in special circumstances. II-In children, thrombophilia and miscellaneous conditions. BMJ 2003;326:93-6.

2 Weiss RB, Tormey DC, Holland JF, Weinberg VE. Venous thrombosis during multimodal treatment of primary breast carcinoma. Cancer Treat Rep 1981;65:677-9.

3 Goodnough LT, Saito H, Manni A, Jones PK, Pearson OH. Increased incidence of thromboembolism in stage IV breast cancer patients treated with a five-drug chemotherapy regimen: a study of 159 patients. Cancer $1984 ; 54: 1264-8$

4 Fisher B, Costantino J, Redmond C, Poisson R, Bowman D, Couture J, et al. A randomized clinical trial evaluating tamoxifen in the treatment of patients with node-negative breast cancer who have estrogen-receptorpositive tumors. N Engl J Med 1989;320:479-84.

5 Thodivil PA, Walsh DC, Kakkar AK. Thromboprophylaxis in the cancer patient. Acta Haematol 2001;106:73-80.

(Accepted 17 July 2003)

\section{A mistake that might have been my last}

In the years 1943, 1944, and 1945 there occurred late spring sub-epidemics of bubonic plague in the city of Chuanchow, in Fujian province in south China. Hospital admissions were between 88 and 69 each year.

I was called to see a patient in the emergency room of the hospital. She was a young woman, semi-comatose with a high fever and a tender swelling in the left inguinal region. The usual practice was to aspirate a speck of fluid from the bubo to confirm the presence of bipolar staining bacilli of plague.

Unwisely, I did not wait for nursing assistance, but instead steadied the lymph node between two fingers and pushed in the aspirating needle. The patient jumped, and the tip of the needle emerged and grazed one of my fingers. The epithelium was broken, but there were no bleeding points.

I consulted a colleague with expertise in tropical medicine. He anaesthetised my finger and excised a wedge of skin that included the entire graze, leaving the wound open to granulate. I may have taken some sulphathiazole for a few days. The patient died within 24 hours.

I learnt that it is rash to undertake a delicate technical procedure in an ill patient without any assistance.

David Landsborough retired medical missionary

We welcome articles up to 600 words on topics such as A memorable patient, A paper that changed my practice, My most unfortunate mistake, or any other piece conveying instruction, pathos, or humour. Please submit the article on http:// submit.bmj.com Permission is needed from the patient or a relative if an identifiable patient is referred to. We also welcome contributions for "Endpieces," consisting of quotations of up to 80 words (but most are considerably shorter) from any source, ancient or modern, which have appealed to the reader. 